\title{
Simplified versus standard regimen forfocal radiofrequency ablation of dysplastic Barrett's oesophagus: a multicentre randomised controlled trial
}

Roos E Pauw', Hannah T Ki.mzli', Raf Bisschops, Carine M Sondermeijer, Arjun D Koch, Paul Didden, AnniekW Gotink, Erik\} Schoon, Wouter L Curvers, Jacques J G H M Berg man, Bas LAM Weusten

\section{Summary}

Background For focal radiofrequency ablation of Barrett's oesophagus, a simplified regimen $(3 \times 15 \mathrm{~J} / \mathrm{cm} .2$, without cleaning) bas proven to be as effective as the standard regimen $\left(2 \times 15 \mathrm{~J} / \mathrm{cm} .2\right.$, followed by cleaning, followed by $\left.2 \times 15 \mathrm{~J} / \mathrm{cm} .{ }^{2}\right)$. However, this simplified regimen seemed to be associated with a higher stenosis rate. Therefore, we

lowered the radiofrequency energyand hypothesised that this new simplified regimen would be as effective and safe as the standard regimen.

Methods This randomised non-inferiority trial included patients with dysplastic Barrett's oesophagus or residual Barrett's oesophagus after endoscopie resection or circurnferential radiofrequency ablation, in five European tertiary referral centres. Patients were randomly assigned $(1: 1)$ to the new simplified regimen $(3 \times 12 \mathrm{~J} / \mathrm{cm} 2$, without cleaning) or the standard regimen, with variable block sizes of four, six, and eight patients, stratified by participating hospital Focal radiofrequency ablation was done every 3 months, up to a maximum of three treatments, until all Barrett's oesophagus was eradicated. The primary outcome was complete endoscopie and histological regression of dysplasia and intestinal metaplasia after two focal radiofrequency ablation treatments, assessed in the intention-to-treat population. Non-inferiority was assessed on the basis of the difference between groups in the median percentage of Barrett's oesophagus surface regression, with a non-inferiority margin of $15 \%$. This study is registered with www.trialregister.nl, number NTR4994, and is completed.

Findings Between March 25, 2015, and July 25, 2016, 84 patients were randomly assigned to treatment: 44 to receive the simplified regimen and 40 to receive the standard regimen. One patient assigned to the simplified regimen and four assigned to the standard regimen were excluded because they weree found not to be eligible; therefore the final intention-to-treat population consisted of 43 patients in the simplified ablation group and 36 in the standard ablation group. Complete endoscopie and histological regression of dysplasia and intestinal metaplasia after two focal radiofrequency ablation treatments was achieved in $32(74 \%, 95 \% \mathrm{Cl} 59-87)$ patients treated with the simplified protocol, versus $30(83 \%$, 95\% $\mathrm{Cl}$ 67-94) patients treated with the standard protocol $(\mathrm{p}=0-34)$. Median Barrett's oesophagus surface regression after two focal radiofrequency ablation sessions was $98 \%$ (IQR 95-100) in the simplified regimen group and 100\% (97-100) in the standard regimen group. The difference between medians was $2 \%(95 \% \mathrm{Cl}-0 \cdot 562$ to $3-162)$; thus the simplified regimen was deemed non- inferior to the standard regimen. Stenoses requiring dilatation were observed in four $(9 \%)$ of 43 patients in the simplified regimen group and four (11\%) of 36 in the standard regimen group. Post-procedural bleeding requiring repeat endoscopy occurred in one (2\%) patient in the simplified ablation group and three $(8 \%)$ patients in the standard ablation group. One patient $(2 \%)$ in the simplified treatment group died 36 days after the second radiofrequency ablation procedure, due to an unknown cause.

Interpretation Based on the results ofthis study, we conclude that the simplified regimen is the preferred regimen for focal radiofrequency ablation ofBarrett's oesophagus.

Introduction

Radiofrequency ablation is an established endoscopie ablation technique for eradication of dysplastic Barrett's oesophagus. For treahnent of Barrett's oesophagus of less than $3 \mathrm{~cm}$ in length, residual islands, tongues, or ablation of the squamocolumnar junction after initia! circumferential radiofrequency ablation, a catheter-based 
focal ablation catheter (Barrx 90 [GI solutions Covidien/Medtronic; Sunnyvale, CA, USA]) is available. The advised ablation regimen in Europe for focal radiofrequency ablation of Barrett's oesophagus consists of two double ablations at $15 \mathrm{~J} / \mathrm{cm} 2$ with a cleaning phase in between. These energy settings were derived from clinical dose escalation studies conducted at the Academie Medica! Center (AMC; Amsterdam, Netherlands) in 2007.12 However, most US centres use a regimen with two ablations at $12 \mathrm{~J} / \mathrm{cm} 2$ with a cleaning phase in between, which originates from a randomised controlled trial in the USA (AIM-dysplasia study).' The AIM-dysplasia study was initiated while the dosimetry studies were ongoing at the AMC. As a result, the first dose-escalation from $2 \times 12 \mathrm{~J} / \mathrm{cm} 2$ to $2 \times 2 \times 12 \mathrm{~J} / \mathrm{cm} 2$, which was evaluated at the AMC, was included in the AIM-dysplasia trial protocol, hut the second step-up from $12 \mathrm{~J} / \mathrm{cm} 2$ to $15 \mathrm{~J} / \mathrm{cm} 2$ was not. Since then, all radiofrequency ablation studies from the USA have used a $2 \times 2 \times 12 \mathrm{~J} / \mathrm{cm} 2$ ablation protocol, while all European radiofrequency ablation studies have used $2 \times 2 \times 15 \mathrm{~J} / \mathrm{cm} 2$

Ablation with the Barrx 90 catheter is relatively easy to perform. However, cleaning the ablation zone and removal of the catheter to clean the electrode, followed by reintroduction for the second ablation pass, is time consuming and may be uncomfortable for the patient. A recent randomised study by our group showed that a

simplified regimen $(3 \times 15 \mathrm{~J} / \mathrm{cm} 2$ without cleaning) was

as effective as the standard regimen $(2 \times 15 \mathrm{~J} / \mathrm{cm} 2$, followed by cleaning, followed by $2 \times 15 \mathrm{~J} / \mathrm{cm} 2)$ for eradication of Barrett's oesophagus islands.' However, in a retrospective series, we observed a relatively high stenosis rate (11\%) when this regimen was applied to the whole Barrett's oesophagus segment, including circumferential ablation of the squamocolumnar

junction.' Reducing the energy settings to $12 \mathrm{~J} / \mathrm{cm} 2$ when using the simplified regimen might be the best

compromise between efficacy and an acceptable stenosis percentage.

The aim of this study was to compare the efficacy and safety of a new simplified regimen with the standard regimen for focal radiofrequency ablation treatment of Barrett's oesophagus in a randomised setting. 
Methods

Study design and participants

Tuis randomised controlled trial was done in five tertiary referral eentres for the treatment of Barrett's oesophagus and related neoplasia in the Netherlands and Belgium: the Academie Medica! Center, Amsterdam, Netherlands; St Antonius Hospita!, Nieuwegein, Netherlands; Erasmus Medica! Center, Rotterdam, Netherlands; Catharina Hospita!, Eindhoven, Netherlands; and the University Hospita! Gasthuisberg, Leuven, Belgium

(appendix p 1). SeeOnlineforappendix

Patients aged 18-85 years were eligible if there was presence of a treatment-naive short segment Barrett's oesophagus eligible for treatment with the Barrx 90 device, or in the case of residual Barrett's oesophagus after initia! (one or two sessions) circum- ferential radiofrequency ablation treatment, endoscopie resection, or a combination thereof. Baseline diagnosis before any treatment had to be Barrett's oesophagus with low-grade dysplasia, high-grade dysplasia, or cancer, confirmed by an expert pathologist in the field of gastrointestinal pathology. If patients had cancer or visible abnormalities, an endoscopie resection was required.

Exclusion criteria were presence of an oesophageal stenosis preventing passage of an endoscope or prior dilation because of a stenosis, oesophageal varices, uncorrectable haemostatic disorders, or use of anti- coagulant therapy that could not be discontinued before radiofrequency ablation. Patients who had undergone previous endoscopie resection were excluded if the resected specimen showed a tumour-positive <leep resection margin (Rl resection), invasion into the <leep submucosa (>500 $\mu \mathrm{m})$, poorly or undifferentiated cancer, presence of lymphovascular invasion, or (for biopsies after endoscopie resection and before radiofrequency ablation) invasive cancer.

The study protocol was approved by the Medical Ethics Committees of all five participating hospitals. All patients gave signed informed consent before participation in the study.

Randomisation and masking

Patients were randomly assigned (1:1) to either the new simplified regimen or the standard regimen during the first focal ablation procedure, using online randomisation software (ProMISe). Randomisation consisted ofvariable block sizes of four, six, and eight patients, stratified by participating hospital. Randomisation was performed by a study nurse or PhD candidate. The allocated radio- frequency ablation regimen was not concealed for endoscopists, data collectors, or patients. The two endoscopists who scored the percentage of Barrett's oesophagus surface regression after two focal radio- frequency ablation treatments were blinded for the regimen used and the centre where the patient was treated.

Procedures

All procedures were done on an outpatient basis under conscious sedation using midazolam or monitored anaesthesia using propofol. Upper endoscopy was done using high-resolution endoscopes with white-light endoscopy and virtual chromoendoscopy (narrow-band imaging, Fuji Intelligent Chromo Endoscopy, or iSCAN). After careful inspection of the Barrett's oesophagus mucosa for the presence of visible lesions or stenosis, the patient was randornly assigned to one of the two ablation regimens. The Barrx 90 device was used for focal radiofrequency ablation treatment. During each focal radiofrequency ablation treatment, the oesophagogastric junction was ablated circumferentially, irrespective of its endoscopie appearance.

For the standard ablation regimen, two ablations with

$15 \mathrm{~J} / \mathrm{cm} 2$ were performed. Thereafter, the coagulum

was removed using the distal rim of the Barrx 90 catheter or forceful spraying of water through a spraying catheter, or both. The Barrx 90 electrode was then cleaned outside the patient, and subsequently the endoscope was reintroduced. All ablation zones were then treated with another two ablations at $15 \mathrm{~J} / \mathrm{cm} 2$ (appendix $\mathrm{p} 1$ ). For the simplified regimen, three

successive ablations at $12 \mathrm{~J} / \mathrm{cm} 2$ were performed while

keeping the catheter in place, without cleaning of the ablated area and electrode in between ablations (appendix p 2).

Additional focal radiofrequency ablation sessions were performed after 3 months until all Barrett's oesophagus was endoscopically eradicated. A maximum of three focal radiofrequency ablation treatments was allowed. After three focal radiofrequency ablation treatments, escape endoscopie resection was performed if there was residual Barrett's oesophagus larger than $5 \mathrm{~mm}$ in diameter. For Barrett's oesophagus areas smaller than $5 \mathrm{~mm}$, argon plasma coagulation was performed at $40 \mathrm{~W}$. Argon plasma coagulation was also allowed after two focal radiofrequency ablation treatments.

All patients received high-dose proton pump inhibitor ( $40 \mathrm{mg}$ twice daily) during the entire treatment phase, with the addition of a histamine2-receptor antagonist at bedtime for 14 days after each treatment, and (in most cases) sucralfate suspension 3-4 times a day. Choice of protonpump inhibitor varied by centre and patient, although most patients were prescribed esomeprazole. For post-treatment pain, patients were allowed to take oral paracetamol or diclofenac suppositories if the pain did not respond to paracetamol.

Patients were asked to complete questionnaires about treatment-related symptoms, including pain in the throat, difficulty swallowing, pain during swallow- ing, pain in the treatment area, and abdominal pain. The severity of pain was recorded for each component using a 10-point scale, resulting in a $0-50$ points scale in total. Symptoms were assessed before radiofrequency ablation treatment, immediately afterwards, and 2 days after treatment.

If all Barrett's oesophagus was endoscopically eradicated, biopsies were taken immediately distal $(<5 \mathrm{~mm})$ to the neosquamocolumnar junction and from four quadrants every $2 \mathrm{~cm}$ of the neosquamous epithelium over the length of the original Barrett's oesophagus segment. When histopathological assessment confirmed the absence of intestinal metaplasia and dysplasia, patients were scheduled for follow-up endoscopy at regular time intervals, depending on the worst histology before radiofrequency ablation.

Biopsies were evaluated for the presence of intestinal metaplasia or dysplasia according to the revised Vienna classification;•biopsies from the neosquamous epithelium were assessed for the presence of subsquamous intestinal metaplasia (ie, buried Barrett's oesophagus). 
The regression percentage was scored by two endo- scopists with experience in radiofrequency ablation treatment of Barrett's oesophagus (BW and RP), who were blinded to the regimen used. The regression percentage was scored as an absolute number, using photos obtained before the first radiofrequency ablation treatment and at endoscopy roughly 3 months after the second radiofrequency ablation treatment. Photos were taken as standard of care in the participating Barrett's oesophagus expert centres, hut this was not done in a standardised way. For cases with a discrepancy in Barrett's oesophagus regression percentage of more than $30 \%$ between endoscopists, a telephone consensus meeting took place.

Outcomes

The primary outcome was complete eradication of dysplasia or intestinal metaplasia after two focal radio- frequency ablation treatments assessed in the intention- to-treat (I'IT) population. Complete eradication was defined as absence of dysplasia and intestinal metaplasia 
in all biopsies taken during the first follow-up endoscopy during which complete endoscopie eradication of Barrett's oesophagus was observed. If patients had endo- scopically visible Barrett's oesophagus after two focal radiofrequency ablation treatments and required additional radiofrequencyablation treatment, no biopsies were obtained and patients were considered to not have achieved complete eradication of dysplasia or intestinal metaplasia after two focal radiofrequency ablation treatments.

In a post-hoc analysis, we analysed the percentage of Barrett's oesophagus surface regression after two focal radiofrequency ablation treatments. We also did a further post-hoc analysis of percentage of Barrett's oesophagus surface regression after two focal radiofrequency ablation treatments, as scored in real time by the treating endoscopist, who was not blinded to the allocated ablation regimen.

The secondary outcomes were: complete eradication of dysplasia or intestinal metaplasia after three focal radiofrequency ablation treatments, with or without escape treatment; total number of focal radiofrequency ablation treatments required to achieve complete eradication of dysplasia or intestinal metaplasia; rate of oesophageal stenosis requiring dilatation; overall complications requmng admission or unplanned endoscopy; median procedure time; and post-procedural pain, measured on a $0-50$ points scale for the severity of symptoms related to radiofrequency ablation treatment. Overall complications requiring admission or unplanned endoscopy were defined as acute if they occurred during the procedure; early if they occurred less than $48 \mathrm{~h}$ after the procedure; and late if they occurred more than $48 \mathrm{~h}$ after the procedure. Complications requiring adrnission or unplanned endoscopy were defined as mild if there was unscheduled hospita! admission, admission was less than 3 days, there was a haemoglobin drop of less than $3 \mathrm{~g} / \mathrm{dL}$, and there was no need for transfusion; moderate if hospita! admission was for 4-10 days, the patient required transfusion of fewer than 4 units of blood, or there was a need for repeat endoscopie treatment, including dilation; and severe if hospita! stay was for more than 10 days, intensive care unit admission was required, surgery was needed, 4 or more units were required for blood transfusion, or, in the case of stenosis, more than five dilatations, stent placement, or incision therapy were needed; and fata! if death was attributable to the procedure and occurred within 30 days of the procedure, or longer with continuous hospitalisation. Only events requiring any intervention were scored,7,'

\section{Statistica! analysis}

The sample size calculation for this study was based on percentage of surface regression of Barrett's oesophagus after two focal radiofrequency ablation sessions, which was estimated to be $96 \%$ based on previous studies with the standard radiofrequency ablation regimen.' 10 However, mistakes were made while calculating the required sample size for this study (see Discussion). First, the percentage of surface regression was not included as an endpoint in the original study protocol. Second, the sample size calculation used to demonstrate non-inferiority with a non-inferiority margin of $-15 \%$ (one-sided a $0-025$, power $90 \%$ ) used a binary variable, whereas percentage of surface regression is a continuous variable. The calculated sample size of 72 patients (36 per group) was therefore not correct and the study might be underpowered to demonstrate non-inferiority of the simplified regimen. Therefore, we included $95 \%$ Cis for all outcomes to put our results into context.

Data analysis was performed using SPSS (version 23). For descriptive statistics, the mean (SD) was used for normally distributed of variables, and the median (IQR)

was used for variables with a skewed distribution. Categorical variables were compared using the $\mathrm{x} 2$ or

Fisher's exact test. Continuous variables were compared using the $t$ test for nonna! distribution or the Mann-Whitney U test for skewed distribution. Differences were considered significant if the $p$ value was 0-05 or less. Tuis study is registered with www.trialregister.nl, number NTR4994.

The trial protocol is available online.

Role of the funding source

There was no funding source for this study. The corresponding author had full access to all the data in the study and had final responsibility for the decision to submit for publication.

\section{Results}

Between March 25, 2015, and July 25, 2016, 84 patients were included. After randomisation, five patients were found to be ineligible, were unable to undergo ablation, or were excluded due to technica! issues and were therefore not included in the final analysis (figure). Five patients. Of the 79 patients who were included in the final intention-to-treat analysis, 43 were randomised to the simplified treatment group, and 36 to the standard treatment group. Patients had a median Barrett's oesophagus length of COM2. Before radiofrequency ablation treatment, 35 (44\%) of 79 patients underwent an endoscopie resection because of a visible lesion $(15$ [42\%] in the standard treatment group vs 20 [47\%] in the simple treatment group). 34 (43\%) of 79 patients underwent primary circumferential radiofrequency ablation treatment before inclusion in the study (14 [39\%] in the standard treatment group vs 20 [47\%] in the simple treatment group). Worst docu- mented histology, based on either biopsies or resection specimen, was low-grade dysplasia in 23 (29\%), high- grade dysplasia in 39 (49\%), and early cancer in $17(22 \%)$ patients. Baseline characteristics are shown in table 1. Two patients treated with the simplified protocol for focal radiofrequency ablation treatment did not finish treatment: one patient because of death 36 days after the second radiofrequency ablation treatment, and one because of psychological problems after a complicated treatment course (this patient achieved complete eradication of dysplasia but not of intestinal metaplasia). Both of these patients were included in the intention-to- treat analysis.

After two focal radiofrequency ablation treatments, median Barrett's oesophagus surface regression was $100 \%$ (IQR 97$100)$ with the standard protocol, compared with a median of $98 \%(95-100)$ following the simplified regimen $(\mathrm{p}=\mathrm{O} \cdot 13)$. The difference between medians was $2 \%(-0.562$ to $3 \cdot 162)$. Since the lower limit of the $95 \% \mathrm{Cl}$ was above the non-inferiority 
margin of $15 \%$, non-inferiority was concluded (table 2).

When using the real-time regression score of the treating endoscopist, median Barrett's oesophagus surface regression was $100 \%$ (IQR 99-100) following two focal radiofrequency ablation treatments using the standard regimen, as well as following treatment with the simplifi.ed regimen $(100 \%, 99-100 ; \mathrm{p}=0-43$; table 2$)$.

In the intention-to-treat analysis, complete eradication of dysplasia after three focal radiofrequency ablation treatments, including escape treatment, was achieved in

42 (98\%; $95 \% \mathrm{Cl} 88-100)$ of 43 patients using the simplified regimen, and in all $36(100 \%$; 90-100) patients using the standard regimen $(\mathrm{p}=1)$.

Complete eradication of dysplasia and intestinal metaplasia was achieved in 36 (84\%; 95\% Cl 69-93) of 43 patients using the simplified regimen, and in 33 (92\%; 78-98) of 36 patients using the standard regimen ( $\mathrm{p}=\mathrm{O} \cdot 33$; table 2). Eight of ten patients with residual intestinal metaplasia had no visible residual Barrett's oesophagus mucosa, but only focal intestinal metaplasia in biopsies obtained just distal to a norrnal-appearing oesophagogastric junction. No additional radiofrequency ablation of the oesophagogastric junction was perforrned in these eight patients because: they had either already been treated with the maximum of three radiofrequency ablation sessions $(n=5)$; because of comorbidity and old age ( $n=1)$; because of the presence of a stenosis $(n=1)$; or patient preference $(n=1)$. The other two patients with residual intestinal metaplasia did not finish the treatment.

In the intention-to-treat analysis, complete eradication of dysplasia or intestinal metaplasia after two focal ablation sessions was achieved in 32 (74\%; 95\% CI 59-87) of 43 patients treated with the simplified proto- col, versus 30 (83\%; 67-94) of 36 patients treated with the standard protocol $(\mathrm{p}=\mathrm{O} \cdot 34$; table 2). All other patients required additional treatment for residual Barrett's mucosa.

Escape treatrnent was performed in $16(37 \%$ CI 23-53) of 43 patients in the simplified treatrnent group and eight (22\%; CI 10-39) of 36 patients in the standard treatrnent group $(\mathrm{p}=0 \cdot 15)$. Escape treatrnent consisted of argon plasma coagulation in 21 (88\%) patients (15 of 16 in the simplified group, six of eight in the standard group), escape endoscopie resection in two (8\%) patients in the standard group, or combination in one (4\%) patient in the simplified group. Endoscopie resection specimens showed squamous epithelium without intestinal metaplasia $(n=1$ in the simplified group), intestinal metaplasia without dysplasia ( $\mathrm{n}=\mathrm{l}$ in the standard group), and Tlm 2 cancer (ie, mucosal cancer infiltrating the lamina propria; $\mathrm{n}=1$ in the standard group). The Tlm2 lesion developed in a patient initially diagnosed with high-grade dysplasia without a visible lesion; however, after one radiofrequency ablation session, a visible lesion (Paris classification type 0-Ilb) was detected and curatively removed. Buried Barrett's oesophagus under the neosquamous epithelium was found in one (1\%) patient in the simplified treatrnent group.

A median of 2 (IQR 1-2) focal radiofrequency ablation treatment sessions was required to achieve complete eradieation of all Barrett's epithelium for both regimens. Oesophageal stenosis requiring dilation therapy developed in four $(9 \%$; $95 \% \mathrm{CI}$ 2. 5-22.1) of 43 patients after focal radiofrequency ablation treatrnent using the simplified regimen, and four $(11 \% ; 95 \% \mathrm{Cl}$

$3 \cdot 1-26 \cdot 1)$ of 36 patients using the standard regimen $(\mathrm{p}=1)$. A median of 2 (IQR 1-3) dilation sessions were required to resolve the stenoses $(2 \cdot 5[\mathrm{IQR} 2 \cdot 0-3 \cdot 0]$ in the simplified treatment group $v s 1 \cdot 5[1 \cdot 0-2 \cdot \mathrm{O}]$ in the standard treatrnent group; $\mathrm{p}=\mathrm{O} \cdot 23$ ).

Complieations leading to additional endoscopy or hospital admission were observed in seven patients in the simplified group and in six in the standard group ( $\mathrm{p}=0-25$; table 3 ). Five of these complications were graded as severe (two in the simplified group $v s$ three in the standard group; $\mathrm{p}=\mathrm{O} \cdot 36$ ). Four patients experienced bleeding a median of 9 (IQR 7-12) days after radio- frequency ablation treatrnent, three of whom were treated with the standard regimen. These patients underwent an upper endoscopy with injection of adrenaline $(n=2)$, adrenaline and clips $(n=1)$, or no intervention $(n=1)$. All four patients were admitted to the hospital for 4 days. One patient treated with the simplified regimen developed a stenosis after the first focal radiofrequency ablation treatrnent. Tuis patient subsequently developed dysphagia requmng dilation therapy. During the second dilatation in a community hospital not participating in this study, a perforation developed, whieh was treated by the placement of a stent and intravenous antibiotics. Tuis patient was adrnitted to hospita \} for 5 days, and 7 days after leaving hospital was readmitted for 9 days because of pain complaints due to stent placement. 4 weeks after the perforation occurred, the stent was removed. Residual Barrett's oesophagus was treated with additional radiofrequency ablation. Following this radiofrequency ablation treatrnent, a few Barrett's oesophagus islands of $3 \mathrm{~mm}$ in diameter were observed. Biopsies showed Barrett's oesophagus without dysplasia. However, the patient refused to undergo another gastroscopy due to psychological complaints that developed after the perforation; this patient therefore failed to achieve complete eradieation of intestinal metaplasia. An endoscopy was performed in one patient because of dysphagia, hut no stenosis was seen. One patient in the simplified regimen group died at home due to an unknown cause, 36 days after the second focal radiofrequency ablation treatrnent.

Median procedure time of the simplified regimen was 13 min (IQR 10-17), compared with 20 min (16-24) for the standard regimen ( $\mathrm{p}<0.0001$; difference in medians 7, 95\% CI 5-9). Median ablation time, measured from introduction until removal of the Bam 90 device, was 7 min (IQR 5-9) for the simplified regimen and $13 \mathrm{~min}$ (IQR 10-16) for the standard regimen $(\mathrm{p}<0-0001$, difference in medians $6 ; 95 \% \mathrm{Cl} 4-8$ ).

Before focal radiofrequency ablation, overall patient discomfort was O (IQR 0--0) on a 0-50 points scale for the severity of symptoms related to radiofrequency ablation treatment (table 4). Directly after radiofrequency ablation, $38 \%$ of patients reported pain, with an overall discomfort of 4 (2-6). At day 2 after radiofrequency ablation, $70 \%$ of patients reported discomfort, these patients had a median score of 4 (3-6), and $48(61 \%)$ of 79 patients took analgesics to relieve the symptoms (paracetamol in most patients). $24(30 \%)$ patients were free of symptoms at day 2. No significant differences in postprocedural pain were found between the two regimens.

At the end of the treatment period, after finishing radiofrequency ablation treatment and escape treatment if necessary, 
complete eradication of dysplasia was achieved in $42(98 \%)$ patients in the simplified treatment group and in $36(100 \%)$ of patients in the standard group; complete eradication of intestinal metaplasia was noted in $36(84 \%)$ and $33(92 \%)$, respectively.

\section{Discussion}

In this randomised trial we showed that a simplified regimen $(3 \times 12 \mathrm{~J} / \mathrm{cm} 2$, without cleaning) was non-inferior to the standard regimen $(2 \times 15 \mathrm{~J} / \mathrm{cm} 2$, followed by cleaning, followed by $2 \times 15 \mathrm{~J} / \mathrm{cm} 2)$ for focal radiofrequency ablation treatment of dysplastic Barrett's oesophagus.

Two flaws in the design of this study carne to our attention only after finishing the trial. First, our sample size calculation was based on Barrett's oesophagus surface regression after two focal radiofrequency ablation sessions, but this parameter was accidentally not included as an endpoint in the original study protocol. This endpoint was evaluated at the end of the study and added as a post-hoc analysis. Second, for our sample size calculation we aimed tocalculate the number of patients required todemonstrate non-inferiority of the simplified regimen compared with the standard regimen. For this, we used the percentage of surface regression of Barrett's oesophagus after two focal radiofrequency ablation sessions as the relevant outcome, and we defined non-inferiority as $15 \%$ difference in Barrett's oesophagus surface regression after two focal radiofrequency ablation sessions. Based on prior studies we know that Barrett's oesophagus surface regression after two focal radiofrequency ablation sessions is $96 \%$ in most patients. We set a $15 \%$ difference as the non-inferiority margin, since regression of $80 \%$ or less would probably need significant additional radiofrequency ablation treatment, whereas patients with more than $80 \%$ Barrett's oesophagus surface regression could be treated with argon plasma coagulation or escape endoscopie resection. However, Barrett's oesophagus surface regression is a continuous outcome and the sample size formula we used was based on binary outcomes, which in hindsight was incorrect. However, the results of our study showed a $95 \% \mathrm{Cl}$ between means of O. 562 and 3-162, and the lower limit of this $95 \% \mathrm{Cl}$ was well above the predefined non- inferiority margin of $15 \%$. These results, in our opinion, 
demonstrate non-inferiority of the simple regimen compared with the standard regimen and we feit it would be artificial to perform a post-hoc sample size calculation.

In addition to Barrett's oesophagus surface regression after two focal radiofrequency ablation sessions, other important outcomes of this study were complete eradication of dysplasia or intestinal metaplasia after two focal radiofrequency ablation sessions. In the majority of radiofrequency ablation studies, most patients require two focal radiofrequency ablation sessions to achieve complete eradication of dysplasia or intestinal metaplasia.'. ${ }^{712} \mathrm{~A}$ significant difference in the number of patients requiring additional radiofrequency ablation after two treatment sessions would therefore be clinically relevant. Since no significant difference in the percentage of patients achieving complete eradication of dysplasia or intestinal metaplasia was found after two sessions, the simplified and standard regimen appeared to be equally effective.

The rates of complete eradication of dysplasia or intestinal metaplasia after two focal radiofrequency ablation sessions reported in this study ( $74 \%$ for the simple regimen and $83 \%$ for the standard regimen), should not becompared with eradication rates mentioned in other publications on radiofrequency ablation, since these generally only report eradication rates at the end of the treatment period. Additionally, since we <lid not obtain biopsies before additional ablation, the rate of complete eradication of dysplasia or intestinal metaplasia after two focal radiofrequency ablation sessions might even be higher than that reported in this study.

After finishing radiofrequency ablation treatment and escape treatment if necessary, complete eradication of dysplasia was achieved in $\mathbf{9 8 \%}$ of patients in the simplified treatment group and in $100 \%$ of patients in the standard treatment group in the intention-to-treat analysis and complete eradication ofintestinal metaplasia and dysplasia was achieved in $84 \%$ and $92 \%$, respectively. These results aresimilar to the results of a large European multicentre study (EURO-II study), in which complete eradication of dysplasia was achieved in $92 \%$ of patients and complete eradication of intestinal metaplasia in $87 \%$ of patients. Four of the centres participating in this randomised trial were also part of the EURO-II study, in which the standard regimen for focal radiofrequency ablation treatment was used. ${ }^{2}$

Complete eradication of intestinal metaplasia was not achieved after two or three focal radiofrequency ablation sessions in ten patients. In eight of these patients, there was no visible Barrett's oesophagus mucosa, but persistent intestinal metaplasia was detected in biopsies obtained just below the neosquamocolumnar junction. However, the clinical relevance of intestinal metaplasia in a normal-appearing neosquamocolumnar junction during endoscopy could be questioned. Intestinal metaplasia in the cardia is found in up to $25 \%$ of the healthy population, and several studies have shown that focal intestinal metaplasia of the cardia often follows a benign course."-" The follow-up of this study is too short to report on the long-term outcomes of these eight patients. Occult intestinal metaplasia in the neosquamous epithelium was found in one (1\%) patient. The genera! fear is that buried Barrett's oesophagus might progress to dysplasia or cancer while remaining endoscopically invisible. However, the incidence of buried Barrett's oesophagus after radiofrequencyablation is reported to be very rare, and our results are in line with

the results of earlier studies.u••"."

Our study did not show a significant difference in complication rate between treatment regimens. The number of stenoses was similar, as was the number of dilatations required to resolve the stenoses. Although this study was not powered to look at differences in complications, these results might have implications for daily practice. The use of a simplified regimen has several advantages regarding the safety profile. Omitting the cleaning step is more practical for the endoscopist, shortens procedure time, and diminishes patient discomfort. Furthermore, the risk of damaging the hypopharynx with the endoscope-mounted Barrx 90 catheter during introduction decreases by $50 \%$ when using the simplified regimen since it only requires one introduction. Although trauma to the hypopharynx or oesophageal wall did not occur in this study, it has been described in up to $10 \%$ of patients during introduction of the endoscope.' Furthermore, a significantly shorter procedure time for the simplified regimen might also result in fewer sedation-related risks. Our overall stenosis rate of $9 \%$ lies above the pooled estimate rate $(6 \%)$ of a recent meta-analysis." However, most of the included studies were retrospective nature and included a heterogeneous population. In studies performed by our group we found a stenosis rate ranging between $0 \%$ and $12 \% .{ }^{\prime} \cdot{ }^{\prime 20}$ Of note, three patients experienced bleeding after treatment with the standard regimen, compared with one patient after treatment with the simplified regimen. However, these numbers are too low to draw conclusions regarding a possible diminished risk of bleeding with the simplified regimen.

All focal radiofrequency ablation treatments in this study were performed using the Barrx 90 catheter. However, various differently sized focal radiofrequency ablation catheters are available-ie, the Barrx 90 Ultra device, the Barrx60device, and the charme!radiofrequency ablation device. Since most published studies on the safety and efficacy of radiofrequency ablation treatment for Barrett's oesophagus used the Barrx 90 device, the ablation regimen recommended for all focal radiofrequency ablation devices is based on previous experience with this device. For uniformity of focal radiofrequency ablation treatment, we advocate the use of the simple regimen studied in this randomised trial in combination with all focal radiofrequency ablation devices. 
Strengths of our study include the randomised design. assessment of all histology specimens before focal radiofrequency ablation treatment by expert gastrointestinal pathologists, and the participation of five European centres with a tertiary referral function for Barrett's oesophagus neoplasia. Furthermore, we ablated the oesophagogastric junction in a circumferential manner during each focal radiofrequency ablation session. In combination with aggressive acid suppression therapy after each focal radiofrequency ablation treatrnent, we fee! that this is the optima! treatment protocol for Barrett's oesophagus with dysplasia. Last, all patients underwent a rigorous biopsy protocol during the first follow-up endoscopy after radiofrequency ablation treatrnent: not only the area directly distal to the neosquamocolumnar junction was biopsied, hut biopsies were also obtained every $2 \mathrm{~cm}$ of the neosquamous epithelium over the length of the original Barrett's oesophagus segment.

Several limitations of this study need to be acknowledged in addition to the sample size calculation. First, we evaluated only short-term efficacy, and therefore we cannot confirm whether the simplified regimen is as durable as the standard regimen used in previous studies.'"' Indeed, a simplified regimen might imply some practical challenges such as avoiding too much overlap in treatrnent zones and avoiding missed areas that cannot be treated by the second pass in the standard regimen. Second, we included only patients in tertiary referral centres, and therefore results of this study might not be extrapolated to common practice. Third, escape therapy was performed for small Barrett's oesophagus areas by means of argon plasma coagulation or escape endoscopie resection in a considerable number of patients. However, this did not affect our primary outcome, since escape therapy was allowed only after two focal radiofrequency ablation treatments. Argon plasma coagulation is a commonly used, cheap, and effective ablation technique for the treatrnent of small Barrett's oesophagus areas." Of note, escape treatment with argon plasma coagulation for small Barrett's oesophagus islands $(<5 \mathrm{~mm})$ was applied in $16(37 \%)$ patients in the simple treatrnent group versus eight (22\%) patients in the standard treatrnent group. For comparison, escape treatrnent using argon plasma coagulation or endoscopie resection was applied in 18\% of patients in the EURO-II study, in which the standard regimen for focal radiofrequency ablation was used." However, in the EURO-II study, escape treatrnent was allowed only after the maximum number of three focal radiofrequency ablation sessions had been applied, whereas escape treatrnent was allowed in our study for small Barrett's oesophagus islands remaining after two focal radiofrequency ablation treatrnents. Although not significantly different, the difference in number of patients requiring escape treatrnent between treatrnent regimens could be explained by the fact that, when using the standard regimen, small areas that might be missed during the first ablation pass are ablated during the second ablation pass. When using the simple regimen, the catheter is kept in place for the three ablations, and small areas of Barrett's oesophagus that are located just adjacent to the catheter can be missed.

In conclusion, although this randomised controlled trial was not correctly powered to assess non-inferiority of a simplified regimen ( $3 \times 12 \mathrm{~J} / \mathrm{cm} 2$, without cleaning) for focal radiofrequency ablation treatment versus the standard regimen $(2 \times 15 \mathrm{~J} / \mathrm{cm} 2$, followed by cleaning, followed by $2 \times 15 \mathrm{~J} / \mathrm{cm} 2)$, the simplified protocol seemed to be non-inferior based on a post-hoc assessment of the percentage of Barrett's oesophagus surface regression after two focal radiofrequency ablation treatments. Since the procedure time was also significantly shorter and the simplified regimen was equally safe, the simplified regimen appears to be the preferred method for focal radiofrequency ablation.

Contributors

RP conceived and designed the study, acquired data, analysed and interpreted data, and critically revised the manuscript for important intellectual content. HK acquired data, drafted the manuscript, analysed and interpreted data, and did statistica! analysis. RB, CS, AK. PD. AG, ES, WC, and JB acquired data and critically revised the manuscript for important intellectual content. BW conceived and designed the study, acquired data, critically revised the manuscript for important intellectual content, and supervised the study.

Declaration of interests

RB reports financial support for research from Fujifilm, Pentax, and Cook Medica!, outside the submitted work; speaker's fee and consultancy for Medtronic, Pentax, and Fujifilm; and consultancy for Boston Scientific. ADK reports financial support for research from

Cook Medica! and Interscope, outside the submitted work; and speakers fee and consultancy for Erbe Elektromedizin. EJS reports speaker's fee and consultancy for Medtronic, Boston Scientific, and Olympus.

JJGHMB reports consultancy for Boston Scientific, Cook Medica!, and Covidien/Medtronic. BLAMW reports financial support for research from C2Therapeutic and Covidien/Medtronic, outside the submitted work. All ether authors deciare no competing interests.

References

1 Gondrie JJ, Pouw RE, Sondermeijer CMT, et al. Effective treatrnent

of early Barrett's neoplasia with stepwise circumferential and focal ablation using the HALO system. Endoscopy 2008; 40: 370-79.

2 Gondrie JJ. Pouw RE, Sondermeijer CMT, et al.

Stepwise circumferential and focal ablation ofBarrett's esophagus with high-grade dysplasia: results of the first prospective series of 11 patients. Endoscopy 2008; 40: 359-69.

3 Shaheen NJ, Sharma P, Overholt BF, et al. Radiofrequency ablation in Barrett's esophagus with dysplasia. N Eng! J Med 2009; 360: 2277-88.

4 van Vilsteren FGI, Phoa KN, Alvarez Herrero L, et al. A simplified regimen for focal radiofrequency ablation ofBarrett's mucosa: a randomized multicenter trial comparing two ablation regimens.

Gastrointest Endosc 2013; 78: 30-38. 
5 Künzli HT, Schölvinclc DW, Phoa KN, et al. Simplified protocol for focal radiofrequency ablation using the HAL090 device: short-term efficacy and safety in patients with dysplastic Barrett's esophagus. Endoscopy 2015; 47: 592-97.

6 Schlemper RJ, Riddell RH, Kato Y, et al. The Vienna classification of gastrointestinal epithelial neoplasia. Gut 2000; 47: 251-25.

7 van Vilsteren FGI, Pouw RE, Seewald S, et al. Stepwise radical endoscopie resection versus radiofrequency ablation for Barrett's oesophagus with highgrade dysplasia or early cancer: a multicentre randomised trial. Gut 2011; 60: 765-73.

8 Pouw RE, Seewald S, Gondrie JJ, et al. Stepwise radical endoscopie resection for eradication ofBarrett's oesophagus with early neoplasia in a cohort of 169 patients. Gut 2010; 59: 1169-77.

9 van Vilsteren FGI, Phoa KN, Alvarez Herrero L, et al. A simplified regimen for focal radiofrequency ablation of Barrelt mucosa using the HAL0(90) System: a randomized multicenter trial comparing two ablation regimens. Gastrointest Endosc 2013; 78:30-38.

10 Pouw RE, Wirths K, Eisendrath P, et al. Efficacy of radiofrequency ablation combined with endoscopie resection for barrelt's esophagus with early neoplasia. din Gastroenterol Hepatol 2010; 8: 23-29.

11 Phoa KN, Pouw RE, Bisschops R et al. Radiofrequency ablation combined with endoscopie resection is highly effective for eradication of early Barrelt's neoplasia: Final results from a large prospective European multicenter study (EURO-II) Gastrointest Endosc 2013; 77: AB137.

12 Phoa KN, Pouw RE, Bisschops R, et al. Multimodality endoscopie eradication for neoplastic Barrelt oesophagus: results of an European multicentre study (EURO-II). Gut 2016; 65: 555-56.

13 Morales TG, Camargo E. Bhattacharyya A, Sampliner RE. Long-term follow-up of intestinal metaplasia of the gastric cardia AmJ Gastroenterol 2000; 95: 1677-80.

14 Fleischer DE, Overholt BF, Sharma VK, et al. Endoscopie radiofrequency ablation for Barrett's esophagus: 5-year outcomes from a prospective multicenter trial. Endoscopy 2010; 42:781-89.

15 Phoa KN, Pouw RE, van Vilsteren FGI, et al. Remission ofBarrett's esophagus with early neoplasia 5 years after radiofrequency ablation with endoscopie resection: a Netherlands cohort study. Gastroenterology 2013;145:96-104.

16 Lyday WD, Corbett FS, Kuperman DA, et al. Radiofrequency ablation ofBarrett's esophagus: outcomes of 429 patients from a multicenter community practice registry. Endoscopy 2010; 42: $272-78$.

17 Gray NA, Odze RD, Spechler SJ. Buried metaplasia after endoscopie ablation ofBarrett's esophagus: a systematic review. AmJ Gastroenterol 2011; 106: 1899-908.

18 Qumseya BJ, Wani S, Desai M, et al. Adverse events after radiofrequency ablation in patients with Barrett's esophagus: a systematic review and meta-analysis. din Gastroenterol Hepatol 2016; 14: 1086-95.e6.

19 Phoa KN, van Vilsteren FGI, Weusten BLAM, etal. Radiofrequency ablation $v s$ endoscopie surveillance for patients with Barrett esophagus and low-grade dysplasia. JAMA 2014; $311: 1209$

20 Pouw RE, Gondrie JJ, Sondermeijer CM, et al. Eradication of Barrelt esophagus with early neoplasia by radiofrequency ablation, with or without endoscopie resection. JGastrointest Surg 2008;12:1627-36

21 Manner H, Rabenstein T, Pech 0, et al. Ablation of residual Barrett's epithelium after endoscopie resection: a randomized long-term follow-up study of argon plasma coagulation

vs. surveillance (APE study). Endoscopy 2014; 46: 6-12. 


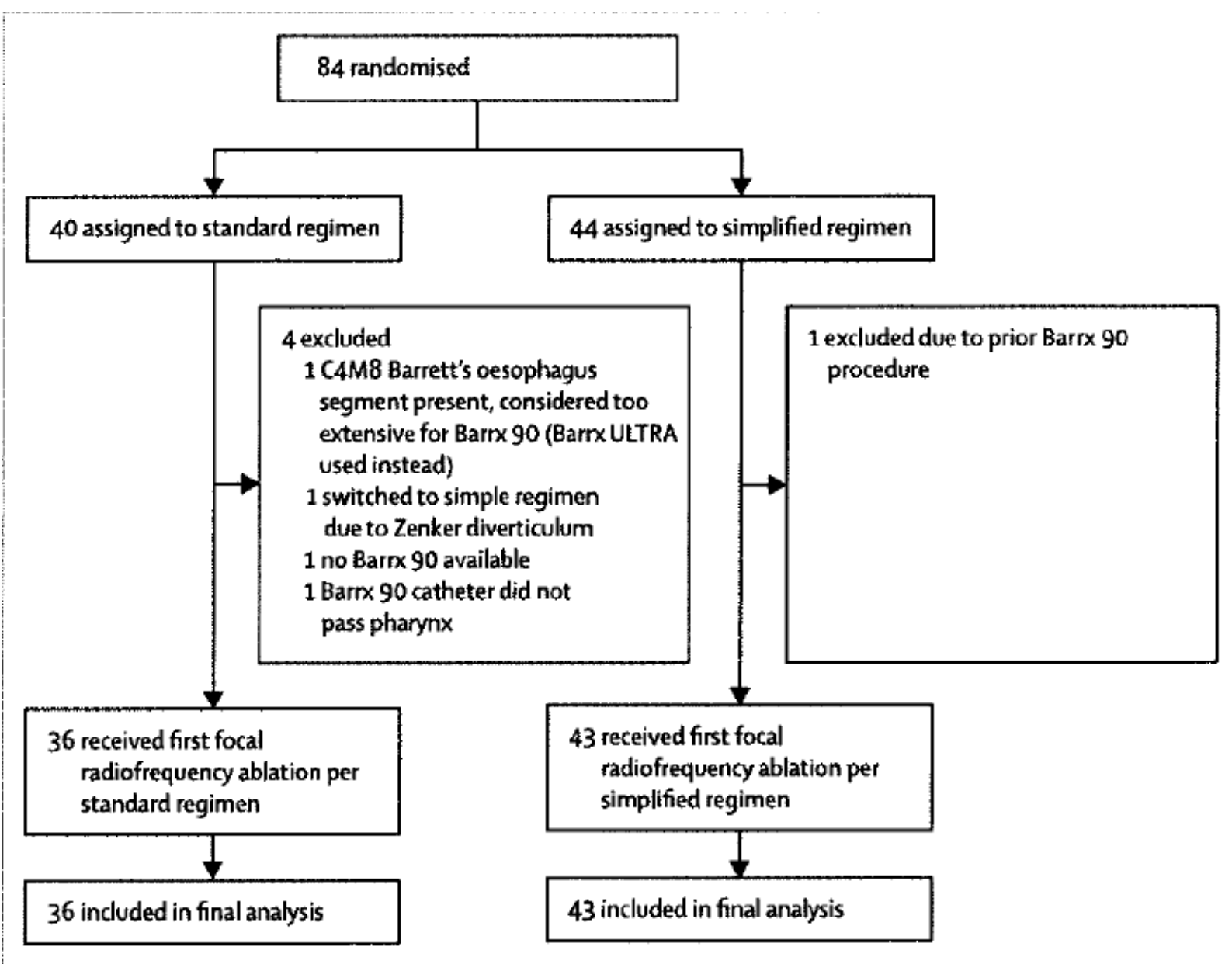




\begin{tabular}{|c|c|c|}
\hline & $\begin{array}{l}\text { Standard regimen } \\
(n=36)\end{array}$ & $\begin{array}{l}\text { Simplified regimen } \\
(n=43)\end{array}$ \\
\hline \multicolumn{3}{|l|}{ Sex } \\
\hline Male & $30(83 \%)$ & $41(95 \%)$ \\
\hline Female & $6(17 \%)$ & $2(5 \%)$ \\
\hline Median age (years) & $66(61-74)$ & $64(52-73)$ \\
\hline Reported use of proton-pump inhibitors & $36(100 \%)$ & $43(100 \%)$ \\
\hline Endoscopic resection before radiofrequency ablation & $15(42 \%)$ & $20(47 \%)$ \\
\hline \multicolumn{3}{|c|}{ Worst documented histology before radiofrequency ablation ${ }^{*}$} \\
\hline Low-grade dysplasia & $12(33 \%)$ & $11(26 \%)$ \\
\hline High-grade dysplasia & $17(47 \%)$ & $22(51 \%)$ \\
\hline Mucosal oesophageal adenocarcinoma & $5(14 \%)$ & $9(21 \%)$ \\
\hline Submucosal oesophageal adenocarcinoma & $2(6 \%)$ & $1(2 \%)$ \\
\hline Barr 360 before radiofrequency ablation & $14(39 \%)$ & $20(47 \%)$ \\
\hline \multicolumn{3}{|c|}{ Barrett's oesophagus length before focal radiofrequency ablation $(\mathrm{cm})$} \\
\hline Circumferential & $0(0-0)$ & $0(0-0)$ \\
\hline Maximum & $2(1-2)$ & $1(1-2)$ \\
\hline \multicolumn{3}{|c|}{ Data are $n(\%)$ or median (IQR). Based on endoscopic resection specimens and biopsies. } \\
\hline
\end{tabular}




\begin{tabular}{|c|c|c|c|}
\hline & $\begin{array}{l}\text { Standard regimen } \\
(n=36)\end{array}$ & $\begin{array}{l}\text { Simplified regimen } \\
(n=43)\end{array}$ & P value \\
\hline $\begin{array}{l}\text { Complete eradication of dysplasia and intestinal } \\
\text { metaplasia after two focal radiofrequency ablation } \\
\text { treatments }\end{array}$ & $30(83 \%)$ & $32(74 \%)$ & 0.34 \\
\hline $\begin{array}{l}\text { Barrett's oesophagus surface regression after } \\
\text { two focal radiofrequency ablation treatments }\end{array}$ & $100 \%(97-100)$ & $98 \%(95-100)$ & 0.13 \\
\hline $\begin{array}{l}\text { Barrett's oesophagus surface regression after } \\
\text { two focal radiofrequency ablation treatments } \\
\text { (as scored real-time by the treating endoscopist) }\end{array}$ & $100 \%(99-100)$ & $100 \%(99-100)$ & 0.43 \\
\hline $\begin{array}{l}\text { Complete eradication of dysplasia and intestinal } \\
\text { metaplasia after three focal radiofrequency ablation } \\
\text { sessions, with or without escape treatment }\end{array}$ & $33(92 \%)$ & $36(84 \%)$ & 0.33 \\
\hline $\begin{array}{l}\text { Focal radiofrequency ablation treatment sessions } \\
\text { needed to achieve complete eradication of } \\
\text { dysplasia and intestinal metaplasia }\end{array}$ & $2(1-2)$ & $2(1-2)$ & 0.5 \\
\hline Stenosis requiring dilation therapy & $4(11 \%)$ & $4(9 \%)$ & 1 \\
\hline $\begin{array}{l}\text { Complications requiring admission or unplanned } \\
\text { endoscopy }\end{array}$ & $7(19 \%)$ & $6(14 \%)$ & 0.25 \\
\hline Procedure time (min) & $20(16-24)$ & $13(10-17)$ & $<0.0001$ \\
\hline Data are $n(\%)$, median (IQR), or mean (SD). & & & \\
\hline
\end{tabular}




\begin{tabular}{|c|c|c|}
\hline & $\begin{array}{l}\text { Standard } \\
\text { regimen } \\
(n=36)\end{array}$ & $\begin{array}{l}\text { Simplified } \\
\text { regimen } \\
(n=43)\end{array}$ \\
\hline Bleeding & $3(8 \%)$ & $1(2 \%)$ \\
\hline Stenosis & $4(11 \%)$ & $3(7 \%)$ \\
\hline Dysphagia without presence of stenosis & 0 & $1(2 \%)$ \\
\hline $\begin{array}{l}\text { Stenosis, subsequently complicated by } \\
\text { perforation due to dilatation }\end{array}$ & 0 & $1(2 \%)$ \\
\hline \multicolumn{3}{|c|}{$\begin{array}{l}\text { No early or acute complications leading to unplanned endoscopy or admission } \\
\text { were reported. }\end{array}$} \\
\hline
\end{tabular}

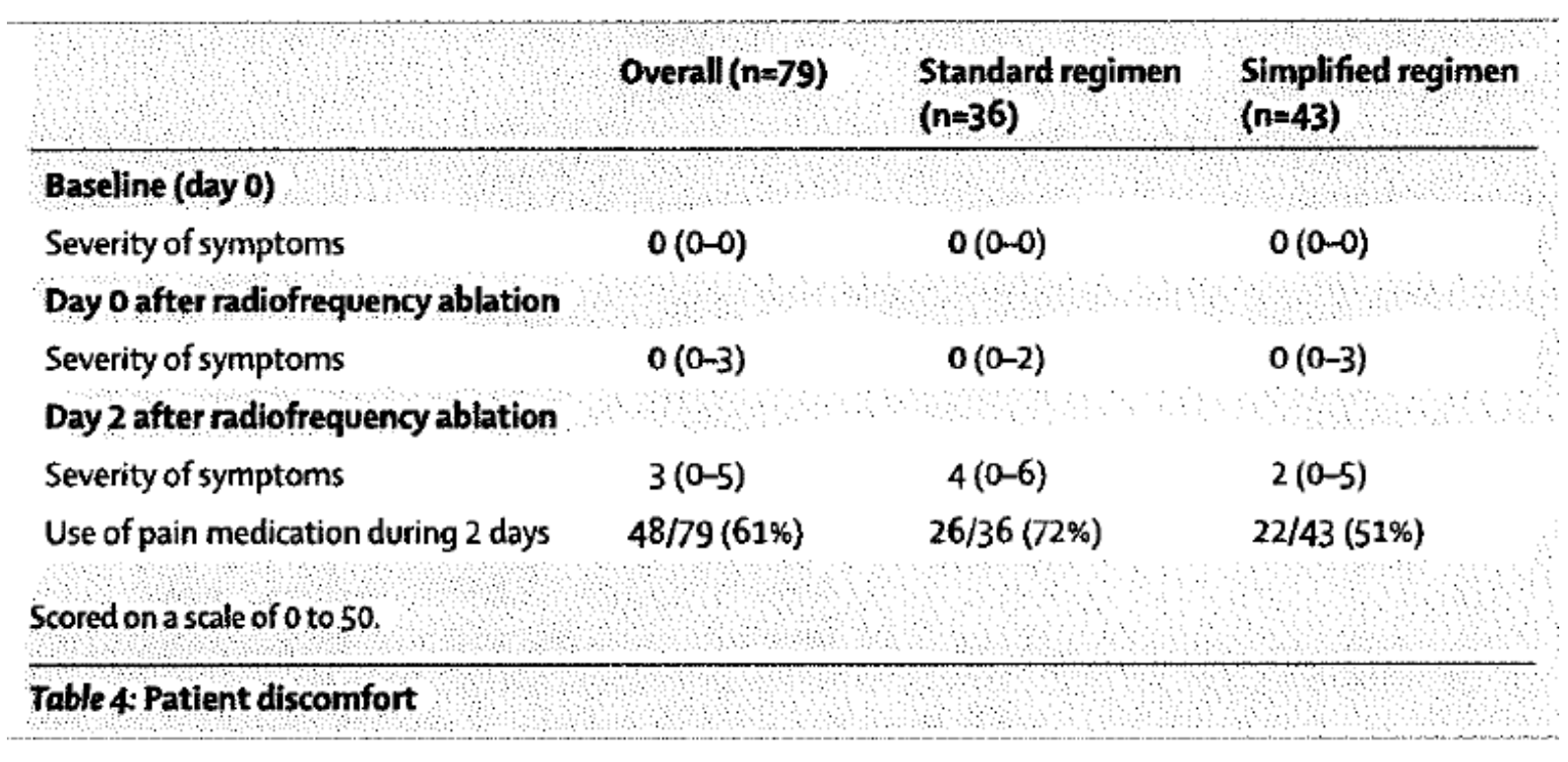

